Review

\title{
Roles of microRNAs in inflammatory bowel disease
}

HyunTaek Jung ${ }^{1 *}$, Jae Seok Kim²*, Keum Hwa Lee ${ }^{3}$, Kalthoum Tizaoui ${ }^{4}$, Salvatore Terrazzino ${ }^{5}$, Sarah Cargnin ${ }^{5}$, Lee Smith $^{6}$, Ai Koyanagi7, , Louis Jacob ${ }^{7,9}$, Han Li ${ }^{10}$, Sung Hwi Hong ${ }^{1}$, Dong Keon Yon ${ }^{11}$, Seung Won Lee ${ }^{12}$, Min Seo Kim ${ }^{13}$, Paul Wasuwanich ${ }^{10}$, Wikrom Karnsakul ${ }^{14}$, Jae Il Shin ${ }^{3 凶}$, Andreas Kronbichler ${ }^{15}$

1. Yonsei University College of Medicine, Seoul, Republic of Korea.

2. Department of Nephrology, Yonsei University Wonju College of Medicine, Wonju, Republic of Korea.

3. Department of Pediatrics, Yonsei University College of Medicine, Seoul, Republic of Korea.

4. Laboratory Microorganisms and Active Biomolecules, Sciences Faculty of Tunis, University Tunis El Manar, Tunis, Tunisia.

5. Department of Pharmaceutical Sciences and Interdepartmental Research Center of Pharmacogenetics and Pharmacogenomics (CRIFF), University of Piemonte Orientale, Novara, Italy.

6. The Cambridge Centre for Sport and Exercise Science, Anglia Ruskin University, Cambridge, CB1 1PT, UK.

7. Research and Development Unit, Parc Sanitari Sant Joan de Déu, CIBERSAM, 08830 Barcelona, Spain.

8. ICREA, Pg. Lluis Companys 23, 08010 Barcelona, Spain.

9. Faculty of Medicine, University of Versailles Saint-Quentin-en-Yvelines, 78000 Versailles, France

10. University of Florida College of Medicine, Gainesville, FL 32610, USA.

11. Department of Pediatrics, Seoul National University Children's Hospital, Seoul National University College of Medicine, Seoul, Republic of Korea.

12. Department of Data Science, Sejong University College of Software Convergence, Seoul, Republic of Korea.

13. Korea University, College of Medicine, Seoul, Republic of Korea.

14. Division of Pediatric Gastroenterology, Hepatology, and Nutrition, Department of Pediatrics, Johns Hopkins University School of Medicine, Baltimore, USA.

15. Department of Internal Medicine IV (Nephrology and Hypertension), Medical University Innsbruck, Innsbruck, Austria.

*These authors contributed equally to this work.

$\triangle$ Corresponding author: Jae Il Shin, M.D., Ph.D. Address: 50 Yonsei-ro, Seodaemun-gu, C.P.O. Box 8044, Department of Pediatrics, Yonsei University College of Medicine, Seoul 03722, Republic of Korea. Tel: +82-2-2228-2050; Fax: +82-2-393-9118; E-mail: shinji@yuhs.ac.

(C) The author(s). This is an open access article distributed under the terms of the Creative Commons Attribution License (https://creativecommons.org/licenses/by/4.0/). See http://ivyspring.com/terms for full terms and conditions.

Received: 2021.02.28; Accepted: 2021.05.08; Published: 2021.05.17

\begin{abstract}
Inflammatory bowel disease (IBD) is a chronic inflammatory disease of the gastrointestinal tract that mainly affects young people. IBD is associated with various gastrointestinal symptoms, and thus, affects the quality of life of patients. Currently, the pathogenesis of IBD is poorly understood. Although intestinal bacteria and host immune response are thought to be major factors in its pathogenesis, a sufficient explanation of their role in its pathophysiologic mechanism has not been presented. MicroRNAs (miRNAs), which are small RNA molecules that regulate gene expression, have gained attention as they are known to participate in the molecular interactions of IBD. Recent studies have confirmed the important role of miRNAs in targeting certain molecules in signaling pathways that regulate the homeostasis of the intestinal barrier, inflammatory reactions, and autophagy of the intestinal epithelium. Several studies have identified the specific miRNAs associated with IBD from colon tissues or serum samples of IBD patients and have attempted to use them as useful diagnostic biomarkers. Furthermore, some studies have attempted to treat IBD through intracolonic administration of specific miRNAs in the form of nanoparticle. This review summarizes the latest findings on the role of miRNAs in the pathogenesis, diagnosis, and treatment of IBD.
\end{abstract}

Key words: inflammatory bowel diseases; Crohn's disease; ulcerative colitis; microRNAs

\section{Introduction}

Inflammatory bowel disease (IBD) is a complex and multifactorial condition characterized by chronic gastrointestinal tract inflammation. There are two main forms of IBD: Crohn's disease (CD) and ulcerative colitis (UC). CD is manifested as patchy transmural inflammatory patterns that affect all layers of the intestinal wall in any portion of the gastrointestinal tract, whereas UC is limited to the innermost layers of the mucosa in the large intestine.
CD and UC mainly affect young people, causing bloody diarrhea, abdominal pain, malabsorption, fatigue, and impaired quality of life [1]. Long-lasting inflammation also increases the risk of colorectal cancer in patients with IBD, which has a mortality rate of $10-15 \%$ [2].

Rapid modernization and urbanization seem to be related to the increasing incidence of IBD, which affects approximately $0.5 \%$ of the general population 
in developed nations [3, 4]; however, the exact pathogenesis of IBD remains poorly understood. Based on speculations, IBD is presumed to be the result of complex interactions among genetic, microbial, and environmental factors [1]. Genomewide association studies (GWASs) have identified the genetic susceptibility loci for IBD, and more than 240 of these loci have been reported [5-8]. Substantial differences have been found between different ethnic groups [9, 10]. Moreover, the existence of IBD susceptibility mutations is not sufficient to explain the entire disruption of intestinal homeostasis; this is because twin studies revealed low concordance between genetic variations and clinical presentation of the disease [11]. The role of the microbiome and lifestyle factors in the pathogenesis of IBD is unquestionable; however, these conditions only cause disease in genetically susceptible individuals [12].

Recent molecular research has shown that microRNAs (miRNAs or miR-) play important roles in the pathophysiology of IBD [13]. miRNAs are evolutionarily conserved, endogenous, singlestranded, non-coding RNAs that bind to the $3^{\prime}$ untranslated region (UTR), 5' UTR, or partially translated region of a target mRNA, inhibiting mRNA translation and blocking its expression [14]. miRNAs are major regulators of cell function and homeostasis, and their abnormal activity has been demonstrated in various diseases, including IBD. Thus, novel treatment options could be developed to alter imbalances in miRNA expression by targeting certain molecular pathways [15]. In this study, we evaluated the role of miRNAs in IBD by examining published results of studies conducted in the past 2-3 years and suggest the clinical potential of miRNAs as biomarkers and/or new therapeutic strategies for IBD.

\section{Biosynthesis of microRNA}

miRNAs are small, 18-21 nucleotide-long, evolutionarily conserved RNA molecules involved in the regulation of gene expression. The primary nuclear transcripts of miRNAs, or pri-miRNAs, are typically a few thousand nucleotides long and are located in introns, exons, or intergenic regions of the human genome. Pri-miRNAs are processed into 70 nucleotide-long, stem-loop structured pre-miRNAs by the RNAse-3-type enzyme Drosha. Pre-miRNAs are then translocated to the cytosol from the nucleus and cleaved by the endoribonuclease Dicer. The remaining miRNA duplex binds with Argonaute to form an RNA-induced silencing complex (RISC). The guidance strand directs the RISC to the complementary region in the 3'-UTR of the target mRNA, and the RISC makes post-transcriptional modifications. When an exact match is found, the target mRNA is degraded; however, when an incomplete match occurs, translation is destabilized [14]. Figure 1 briefly summarizes the biosynthesis of miRNAs and their mechanisms of action.

The seed region responsible for identifying target mRNAs is a 6 nucleotide-long sequence from the 2 nd to the 7 th nucleotide of the miRNA. Based on similar seed sequences, miRNAs are classified into miRNA families. Members of a given miRNA family share almost the same pool of mRNAs as their target as they possess the same seed region. Numerous miRNAs form clusters in the genome and are transcribed as one common pri-miRNA; therefore, their transcription is regulated simultaneously. Notably, the role of miRNAs is redundant; multiple different miRNAs can control a single mRNA, and a given miRNA can inhibit numerous mRNAs. miRNA regulation does not act as a switch; rather, it functions as a synchronized tuner of gene expression [16].

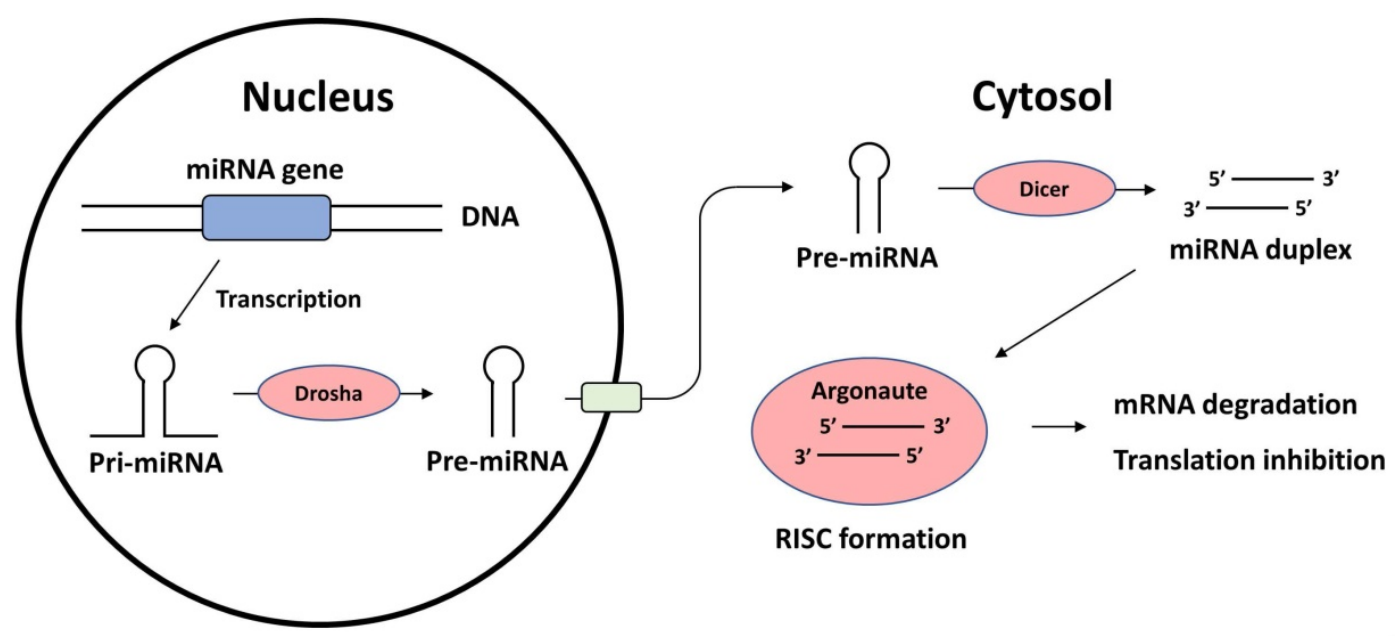

Figure 1. Biosynthesis of miRNA and the mechanism of action. miRNA: microRNA; RISC: RNA-induced silencing complex. 


\section{Homeostasis}

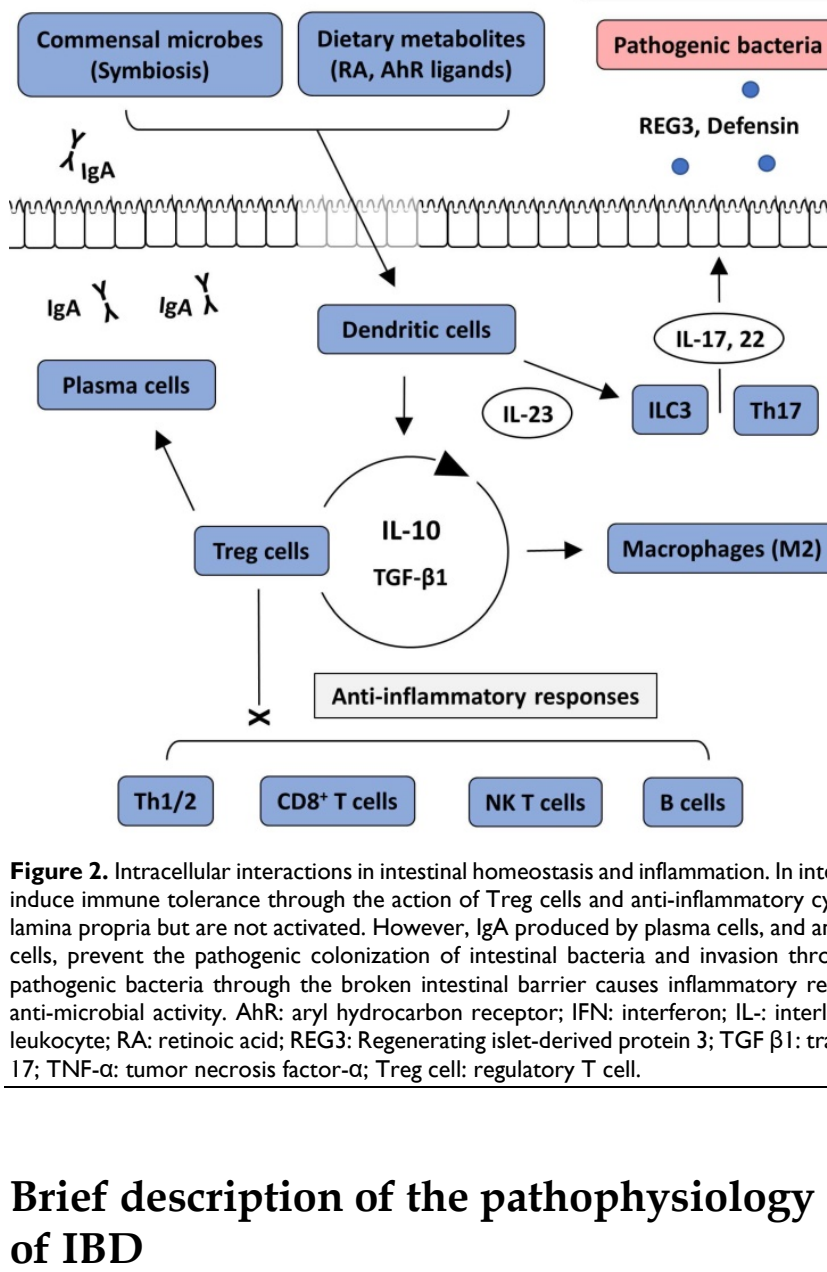

Commensal bacteria are essential components in the maintenance of intestinal homeostasis. They provide essential nutrients, assist in energy metabolism, and suppress the growth of pathogenic bacteria by competitive inhibition in the intestine [17]. Nevertheless, they can cause opportunistic infections and are under the surveillance of host immunity. Consequently, the human immune system has adapted to maintain a commensal relationship by establishing a balance between immune suppression and activation. The intestine is continuously exposed to large amounts of toxins and antigenic proteins from ingested foods. Therefore, a high level of immune tolerance is essential for intestinal homeostasis [18]. However, well-controlled immune responses can be aberrantly activated by altered states of intestinal bacteria or the intestinal barrier, which are two important factors that maintain immune tolerance. From our perspective, a disruption in physiological balance can result in the development of IBD (Figure 2).

Many immune cells, such as neutrophils, dendritic cells, macrophages, and lymphoid cells,
Inflammation

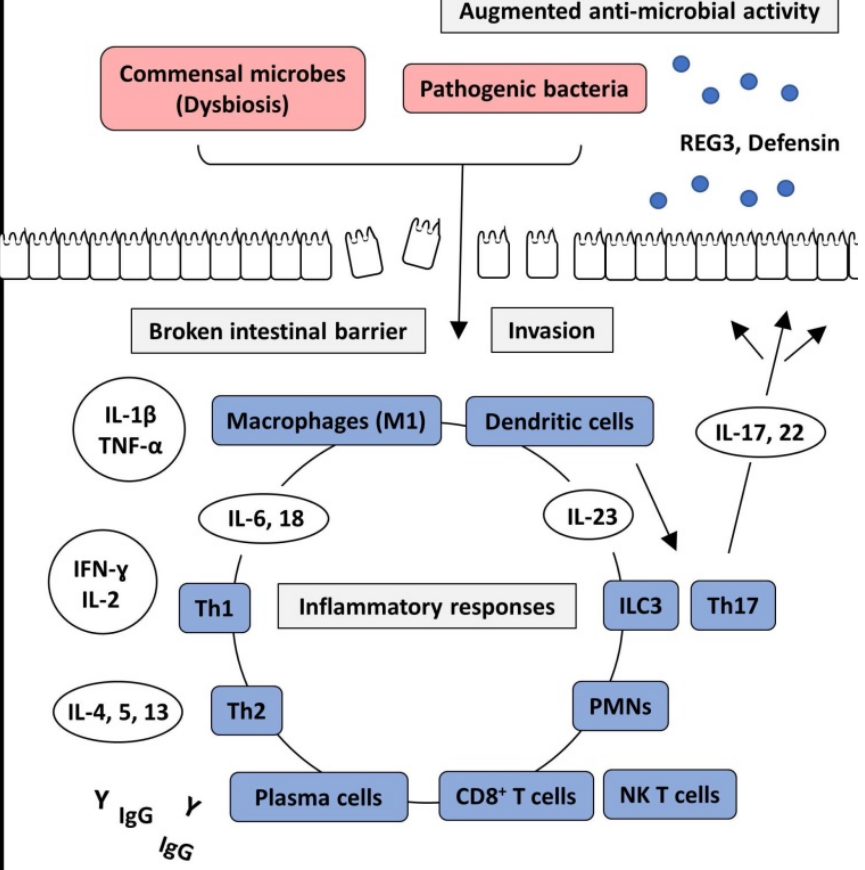


prevents bacteria from invading the intestinal epithelium, and suppress the inflammatory reaction of the intestinal barrier against commensal bacteria [23]. Retinoic acid, a metabolite of vitamin A, is an important nutritional factor that is closely related to the immune response. Retinoic acid plays an important role in maintaining intestinal homeostasis, specifically inducing intestinal immune tolerance through the actions of $\mathrm{CD}_{103^{+}}$dendritic cells and innate lymphoid cells (ILCs)3 [24]. However, based on a recent study, commensal bacteria reduced retinoic acid and IL-22 by inhibiting retinol dehydrogenase 7 (Rdh7) in intestinal epithelial cells. Consequently, the anti-microbial reaction was downregulated, and a symbiotic state could be maintained [25]. Such findings indicate that retinoic acid has both tolerogenic capacity and antimicrobial activity. Thus, intestinal immune tolerance does not simply mean a decrease in intestinal immunity, but rather a balanced state in which anti-inflammatory and anti-microbial activities coexist. The aryl hydrocarbon receptor $(\mathrm{AhR})$ is a transcription factor that induces anti-inflammatory reactions at the gene level. Previous studies have indicated that AhR reduces intestinal inflammation through anti-inflammatory mediators, such as IL-10 and IL-22. Several AhR ligands exist in the intestine, the majority of which are metabolites of commensal bacteria. Therefore, intestinal microbiota contributes to AhR-induced immunomodulation [26, 27]. Taken together, host immunity has dual effects on the intestinal microbiota to maintain intestinal homeostasis. The intestinal immune system should maintain an appropriate balance between inflammatory and anti-inflammatory responses. Dysbiosis, which is an imbalance in microbes in the intestine, disrupts intestinal homeostasis and should thus be considered as a major cause of IBD [28].

The intestinal epithelium is one of the key components that sustain homeostasis of the intestine. The membranous barrier separates intestinal germs from the submucosal lymphoid tissue to prevent inflammatory responses and integrates molecular signals from dietary metabolites, commensal bacteria, and pathogens to regulate immune reactions [29]. Resident lymphoid cells, such as NK T cells, $\gamma \delta$ T cells, ILCs, intraepithelial lymphocytes, and $\mathrm{CD}^{+}$ mucosal-associated invariant $\mathrm{T}$ cells produce IL-6, IL-17, and IL-22 to activate the signal transducer and activator of transcription (STAT) 3 pathway in response to disruption of the intestinal barrier. The STAT3 pathway promotes the recovery of barrier function and increases mucus secretion and epithelial repair [30]. Macrophages secrete type I interferon (IFN) and activate STAT1 and STAT2 pathways to prevent epithelial apoptosis, promote epithelial differentiation, and maintain the integrity of the intercellular barrier [31]. $\mathrm{CD}^{+} \mathrm{T}$ cells produce IL-17 and IL-22 in response to IL-23 and serum amyloid A, which results in the activation of the STAT3 pathway in the epithelium [32]. These interactions enhance the intestinal barrier against pathogenic luminal bacteria.

When the mucus barrier is broken, permeability through the epithelium increases. These situations are mostly due to the dysfunction of the mucosal barrier itself, or inflammatory damage. Mucous membrane disorder, tight junction disorder, increased intestinal permeability, and increased binding of bacteria to epithelial cells can induce intestinal inflammation. Penetration of intestinal bacteria, including intestinal microbiota, into the lamina propria leads to recognition by phagocytes and the production of inflammatory cytokines by macrophages. Macrophages secrete IL-1 $\beta$ to promote the differentiation of $\mathrm{CD}^{+} \mathrm{T}$ cells into $\mathrm{T}$ helper 17 (Th17) cells and the production of IFN- $\gamma[33,34]$. Damaged epithelium releases IL-18, which disrupts goblet cell maturation and impairs its function, leading to further damage of the membranous barrier [35]. Macrophages enhance the survival of pro-inflammatory $\mathrm{T}$ cells and stimulate epithelial apoptosis through the tumor necrosis factor-a (TNF-a) signaling pathway [36, 37]. IL-6 from macrophages and granulocyte-macrophage colony-stimulating factor (GM-CSF) from ILCs recruit polymorphonuclear leukocytes, which secrete pro-inflammatory cytokines for pathogenic $\mathrm{T}$ cell responses [38-40]. Repeated cycles of dysregulated immune responses result in chronic intestinal injury and fibrotic remodeling in patients with IBD [41].

IBD is a multifactorial disease. Although dysbiosis of commensal bacteria and breakdown of the intestinal barrier are considered as major pathological mechanisms in the development of IBD, other important factors such as genetic aberrations also contribute to its development. In this regard, miRNAs are an emerging risk factor for IBD. miRNAs are involved in the maintenance of intestinal barrier integrity, as well as a large number of intracellular signaling pathways involved in inflammatory or anti-inflammatory actions [42].

\section{Role of miRNAs in the pathophysiology of IBD}

miRNAs have been reported to be associated with several pathophysiological factors of IBD. miRNAs affect the intestinal barrier and inflammatory reactions through various pathological mechanisms. The pathogenic roles of miRNAs in IBD are summarized in Table $\mathbf{1}$. 
Table 1. Roles of miRNAs in the pathogenesis of inflammatory bowel disease

\begin{tabular}{|c|c|c|c|}
\hline miRNAs & Targets & Functions & Ref. \\
\hline \multicolumn{4}{|c|}{ miRNAs that weaken the intestinal barrier } \\
\hline $\operatorname{miR}-21$ & $\begin{array}{l}\text { PTEN/PI3K/Akt } \\
\text { pathway }\end{array}$ & Increases the paracellular permeability of the intestinal epithelium & [49] \\
\hline $\operatorname{miR}-122 a$ & EGFR & Enhances the expression of zonulin and increases epithelial permeability & [48] \\
\hline miR-191a, -212 & $\mathrm{ZO}-1$ & Reduce the expression of ZO-1 & {$[44,45]$} \\
\hline miR-675 & Cadherin E, ZO-1 & Destabilizes the mRNA of cadherin E and ZO-1 & [46] \\
\hline $\operatorname{miR}-874$ & Aquaporin 3 & Decreases the expression of aquaporin 3 & [47] \\
\hline \multicolumn{4}{|c|}{ miRNAs that strengthen the intestinal barrier } \\
\hline $\operatorname{miR}-93$ & PTK6 & Reduces the expression of PTK6, and its downregulation attenuates epithelial injury & [51] \\
\hline $\operatorname{miR}-200 b$ & c-Jun, MLCK & Decreases epithelial damage induced by TNF- $\alpha$ in the intestinal epithelium & [50] \\
\hline \multicolumn{4}{|c|}{ miRNAs that increase inflammation } \\
\hline miR-21 & MIP2, TNF-a & Increases the level of MIP2 and TNF-a & [52] \\
\hline miR-124 & AhR & Suppresses AhR expression and increases pro-inflammatory cytokine production & [53] \\
\hline \multicolumn{4}{|c|}{ miRNAs that decrease inflammation } \\
\hline miR-10a & IL-12/23p40 & Downregulates the expression of IL-12/23p40 and Th1/Th17 cell responses & [56] \\
\hline miR-141 & CXCL12 $\beta$ & Inhibits CXCL12 $\beta$-mediated leukocyte migration & [57] \\
\hline $\operatorname{miR}-320$ & NOD2 & Decreases the expression of NOD2 & [55] \\
\hline \multicolumn{4}{|c|}{ miRNAs that enhance autophagy } \\
\hline $\operatorname{miR}-346$ & $\begin{array}{l}\text { Vitamin-D receptor, } \\
\text { GSK3B }\end{array}$ & Downregulates the expression of GSK3B, which increases ATG16L1 & {$[62]$} \\
\hline miR-665 & XBP1, ORMDL3 & Represses XBP1 and ORMDL3 expression & [63] \\
\hline \multicolumn{4}{|c|}{ miRNAs that inhibit autophagy } \\
\hline miR-20a & ATG16L1, SQSTM1 & Downregulates BECN1, ATG16L1, and SQSTM1 & [64] \\
\hline miR-30C & ATG5, ATG16L1 & Reduces the level of ATG5 and ATG16L1 & {$[65]$} \\
\hline miR-93, -106B & ATG16L1, PTEN & Suppress ATG16L1 expression and PTEN activity & {$[66,67]$} \\
\hline miR-122 & NOD2, NF-кB & Inhibits NOD2 activity and increases NF-kB & [69] \\
\hline $\operatorname{miR}-130 \mathrm{a}$ & p-mTOR & Increases the level of p-mTOR & [71] \\
\hline miR-132, -223 & FOXO3a & Downregulate FOXO3a, which enhances NF-kB signaling & [72] \\
\hline miR-142-3p & ATG16L1 & Decreases ATG16L1 mRNA and protein levels & [68] \\
\hline miR-146b & SIAH2, FOXO3 & Decreases SIAH2 and FOXO3 expression and activates the NF-kB pathway & [74] \\
\hline miR-155 & SHIP-1, FOXO3a & Increases Akt activity by decreasing SHIP-1, downregulates FOXO3a and enhances the NF-kB pathway & {$[75,76]$} \\
\hline miR-192 & NOD2, NF-кB & Downregulates NOD2 expression and inhibits NF-KB activity & [70] \\
\hline miR-196 & LC3-II & Inhibits the accumulation of LC3-II & [77] \\
\hline $\operatorname{miR}-320$ & NOD2 & Reduces NOD2 expression & [55] \\
\hline
\end{tabular}

\section{miRNAs and the intestinal barrier in IBD}

Disrupted intestinal membranes are one of the most significant factors in the pathogenesis of IBD. TNF-a is known to be a major pro-inflammatory cytokine in the pathogenesis of IBD. Therefore, several in vitro experiments have been conducted using intestinal epithelial cells to induce injury by TNF-a [43]. miR-191a and miR-212 are known to damage intestinal barriers. In fact, in vitro studies have shown that their mimics downregulate the expression of zonula occludens (ZO)-1, one of the major components of the tight junction between the intestinal epithelium [44, 45]. By measuring the binding of miR-675 in colon cells in vitro, Zou et al. found that miR-675 destabilized the mRNA of ZO-1 and cadherin E, causing reduced expression of key molecules for intercellular tight junctions [46]. Aquaporin 3 is another important protein in the intestinal membrane. The overexpression of miR-874 in vitro induced by pre-miR-874 transfection in intestinal epithelial cells was demonstrated to decrease the expression of aquaporin 3 [47]. Epidermal growth factor receptor (EGFR) was identified as the target gene of miR-122a. The overexpression of miR-122a was found to increase zonulin expression and intestinal permeability [48]. Further, the overexpression of miR-21 in vitro was found to cause an increase in intestinal barrier defects and was suggested to target the phosphatase and tensin homolog (PTEN)/PI3K (phosphoinositide 3-kinase)/Akt signaling pathway to enhance the paracellular permeability of the intestinal epithelium [49]. As the miR-21 mimic suppressed the level of PTEN and increased the level of phospho-Akt (p-Akt) in vitro, Zhang et al. reported the impairing of intestinal permeability through the PTEN/PI3K/Akt pathway as a potential mechanism [49].

Some miRNAs have been reported to strengthen the intestinal barrier. Transfection of the miR-200b precursor in TNF-a-treated intestinal epithelium in vitro inhibited the damage to trans-epithelial electrical resistance and intercellular tight junctions. Further, c-Jun and myosin light chain kinase (MLCK) were demonstrated to be the targets of miR-200b [50]. Haines et al. revealed that silencing the expression of protein tyrosine kinase 6 (PTK6) with miR-93 in the intestinal epithelium increased the resistance to TNF-a-induced injury [51]. 


\section{miRNAs and immune response in IBD}

miRNAs are known to contribute to the immunological reactions that lead to IBD. Shi et al. compared miR-21 knockout mice to wild-type mice after the induction of intestinal damage by dextran sulfate sodium (DSS); miR-21 knockout mice demonstrated reduced weight loss, intestinal inflammation (confirmed by histopathology), serum leukocyte levels, and TNF- $\alpha$ and macrophage inflammatory protein 2 (MIP2) levels in colon culture supernatants compared to wild-type mice [52]. miR-124 was reported to target the 3'-UTR of AhR to suppress its expression in Caco-2 cells and HT-29 cells in vitro [53]. Intracolonic administration of miR-124 inhibitors and precursors to 2,4,6-trinitrobenzen acid (TNBS)-induced colitis mice was found to alleviate and aggravate intestinal inflammation, respectively [53].

Some miRNAs have been reported to suppress intestinal inflammation. Nucleotide-binding oligomerization domain-containing protein 2 (NOD2) is one of the genes clearly associated with CD [54]. In fact, Pierdomenico et al. reported its relationship with miR-320 [55]. HT29 cells transfected with exogenous miR-320 displayed a downregulated expression of NOD2, while miR-320 inhibitor increased NOD2 expression as well as its downstream signaling pathway, leading to nuclear factor $\mathrm{\kappa B}(\mathrm{NF}-\mathrm{kB})$ and inflammatory cytokines [55]. Wu et al. used human monocyte-derived dendritic cells and $\mathrm{CD}^{+} \mathrm{T}$ cells isolated from the peripheral blood of IBD patients treated with the miR-10a precursor. The transfection caused decreased expression of IL-12/23p40 (p40 subunit of IL-12 and IL-23) in dendritic cells and suppression of IFN- $\gamma$, TNF-a, and IL-17A in CD4 $4^{+} \mathrm{T}$ cells, suggesting inhibited differentiation into Th1 and Th17 cells [56]. C-X-C motif chemokine ligand $12 \beta$ $(\mathrm{CXCL12} \beta)$ is an important mediator of leukocyte migration. Huang et al. used TNBS-induced colitis mice, IL-10 knockout colitis mice, and colon biopsy samples of $\mathrm{CD}$ patients to identify an inverse relationship between miR-141 expression and CXCL12 $\beta$ levels [57]. Intracolonic administration of miR-141 precursors and inhibitors to both TNBS-colitis mice and IL-10 knockout mice alleviated and aggravated colitis, respectively [57].

\section{miRNAs and autophagy in IBD}

Autophagy is a cellular response that degrades and recycles internal structures, such as organelles and macromolecules. Therefore, its purpose is to maintain cell homeostasis and regulate inflammatory responses [58]. Defects in autophagy can cause epithelial dysfunction and immune disruption, which play important roles in the pathogenesis of IBD [59].
Recent studies suggest that miRNAs target autophagy-susceptibility genes to regulate intestinal autophagy and control intestinal inflammation in IBD [60]. ATG16L1 is an autophagy-related gene that forms autophagosomes during autophagy [61]. miR-346 was reported to downregulate the expression of the vitamin D receptor, glycogen synthase kinase 3 beta (GSK3B), to increase the level of ATG16L1 in colon biopsy samples of IBD patients [62]. miR-665 represses X-box binding protein 1 (XBP1) and ORMDL sphingolipid biosynthesis regulator 3 (ORMDL3), which also stimulates autophagy [63].

Many miRNAs are known to inhibit autophagy. miR-20a downregulates Beclin 1 (BECN1), ATG16L1, and sequestosome 1 (SQSTM1), which collectively slows down autophagy [64]. miR-30C [65], miR-93, miR-106B [66, 67], and miR-142-3p [68] also target the expression of ATG16L1 to prevent autophagy. miR-122 [69], miR-192 [70], and miR-320 [55] were found to decrease the activity of NOD2 to block autophagy. miR-130a increases the level of phosphorylated mammalian target of rapamycin (p-mTOR) [71], while miR-132, miR-223, miR-146b, and miR-155 reduce Forkhead box class O3 (FOXO3 or FOXO3a) to inhibit autophagy [72-76]. miR-196 blocks the accumulation of the lipid-modified form of microtubule-associated protein 1A/1B-light chain 3 (LC3-II) to prevent autophagy [77].

\section{Role of miRNAs in IBD diagnosis}

Diagnosis and evaluation of IBD have always been challenging. IBD is diagnosed based on clinical manifestations and endoscopy with histopathological examination [78]; however, various clinical manifestations make diagnosis difficult, and endoscopy with histopathology requires the expertise of clinicians [79]. As miRNAs are known to be associated with the pathogenesis of IBD, several studies have suggested that miRNAs are non-invasive and inexpensive biomarkers. A list of possible candidates is provided in Table 2.

\section{miRNA signature from the colon tissues of IBD patients}

Some studies found abnormal elevation of miRNA levels in the mucosal tissues of UC patients compared to those of healthy controls. In fact, $\mathrm{Wu}$ et al. found that miR-16, miR-21, miR-23a, miR-24, miR-29a, miR-126, miR-195, and let-7f were upregulated in active UC patients compared to healthy controls [80]. By comparing the colonic mucosa of UC patients and healthy controls, Fasseu et al. showed that miR-7, miR-26a, miR-29a, miR-29b, miR-31, miR-126* (the complement of miR-126), miR-127-3p, miR-135b, and miR-324-3p were 
increased in the inflamed mucosa of UC patients [81]. Takagi et al. found increased levels of miR-21, miR-155, miR-923, let-7a, let-7c, let-7d, and let-7g in colon biopsy specimens of active UC patients compared to healthy controls [82]. miR-126 [83] and miR-150 [84] were elevated in the colonic mucosa of patients with active UC compared to controls. Frozen biopsy samples of distal colectomy from UC patients were found to have significant increases in miR-31, miR-146a, miR-206, and miR-424 levels [85]. Coskun et al. identified increases in miR-20b, miR-26b, miR-98, miR-99a, and miR-203 levels in colon biopsy samples of active UC patients compared to healthy controls, inactive UC patients, and CD patients [86].

Table 2. miRNA signatures in inflammatory bowel disease

\begin{tabular}{|c|c|c|c|}
\hline $\begin{array}{l}\text { Sample } \\
\text { type }\end{array}$ & Expression & miRNAs & Ref. \\
\hline \multicolumn{4}{|c|}{ Ulcerative colitis vs. healthy controls } \\
\hline \multirow[t]{2}{*}{$\begin{array}{l}\text { Mucosal } \\
\text { tissue }\end{array}$} & Upregulated & $\begin{array}{l}\text { miR-7, miR-16, miR-20b, miR-21, miR-23a, } \\
\text { miR-24, miR-26a, miR-26b, miR-29a, miR-29b, } \\
\text { miR-31, miR-98, miR-99a, miR-126, miR-126*, } \\
\text { miR-127-3p, miR-135b, miR-146a, miR-150, } \\
\text { miR-155, miR-195, miR-203, miR-206, } \\
\text { miR-324-3p, miR-424, miR-923, let-7a, let-7c, } \\
\text { let-7d, let-7f, let-7g }\end{array}$ & [80-86] \\
\hline & Downregulated & $\begin{array}{l}\text { miR-141, miR-188-5p, miR-192, miR-215, } \\
\text { miR-320a, miR-346, miR-375, miR-422b }\end{array}$ & $\begin{array}{l}{[80,81,} \\
87]\end{array}$ \\
\hline $\begin{array}{l}\text { Peripheral } \\
\text { blood }\end{array}$ & Upregulated & $\begin{array}{l}\text { miR-16, miR-19a, miR-21†, miR-28-5p, } \\
\text { miR-101, miR-103-2, miR-142-5p, miR-151-5p, } \\
\text { miR-155, miR-188-5p, miR-199a-5p, miR-223, } \\
\text { miR-340, miR-362-3p, miR-375, miR-378, } \\
\text { miR-422a, miR-494, miR-500, miR-501-5p, } \\
\text { miR-532-3p, miR-769-5p, miR-874, } \\
\text { miRplus-E1271 }\end{array}$ & $\begin{array}{l}{[88,} \\
98-100]\end{array}$ \\
\hline & Downregulated & miR-21†, miR-31, miR-146a, miR-505 & {$[88,99]$} \\
\hline \multicolumn{4}{|c|}{ Crohn's disease vs. healthy controls } \\
\hline \multirow[t]{2}{*}{$\begin{array}{l}\text { Mucosal } \\
\text { tissues }\end{array}$} & Upregulated & $\begin{array}{l}\text { miR-9, miR-21, miR-22, miR-26a, miR-29b, } \\
\text { miR-29c, miR-30b, miR-31, miR-34c-5p, } \\
\text { miR-101, miR-106a, miR-106b, miR-126*, } \\
\text { miR-127-3p, miR-130a, miR-133b, miR-146a, } \\
\text { miR-146b-5p, miR-150, miR-155, miR-181c, } \\
\text { miR-196, miR-196a, miR-206, miR-324-3p, } \\
\text { miR-375, miR-424 }\end{array}$ & $\begin{array}{l}{[66,77,} \\
81,85, \\
88]\end{array}$ \\
\hline & Downregulated & miR-7, miR-375 & {$[88,89]$} \\
\hline $\begin{array}{l}\text { Peripheral } \\
\text { blood }\end{array}$ & Upregulated & 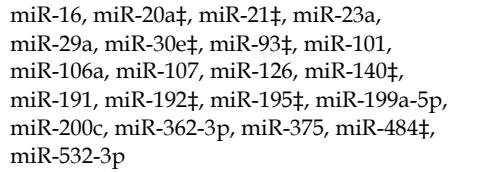 & $\begin{array}{l}{[88,98,} \\
101]\end{array}$ \\
\hline & ate & miR-21, miR-31, miR-146a, miR-155 & \\
\hline
\end{tabular}

*Complementary miRNA, †Upregulation from [98] and downregulation from [88], \$From pediatric patients [101].

Decreased levels of miR-188-5p, miR-215, miR-320a, and miR-346 were observed in the inflamed mucosa of UC patients compared to controls [81]. miR-141 was found to be downregulated in the colonic mucosa of patients with active UC compared to healthy controls [87]. miR-192, miR-375, and miR-422b were downregulated in colon biopsy samples from patients with active UC [80].

The mucosal tissue samples of patients with active $C D$ had aberrant expression of miRNAs compared to controls. Fasseu et al. reported that
miR-9, miR-21, miR-22, miR-26a, miR-29b, miR-29c, miR-30b, miR-31, miR-34c-5p, miR-106a, miR-126*, miR-127-3p, miR-130a, miR-133b, miR-146a, miR-146b-5p, miR-150, miR-155, miR-181c, miR-196a, miR-324-3p, and miR-375 were elevated in the inflamed mucosa of CD patients relative to levels in healthy controls [81]. Further, Lin et al. showed that miR-31, miR-146a, miR-206, and miR-424 were increased in frozen biopsy samples of $\mathrm{CD}$ patients compared to controls, similar to that found in UC patients [85]. miR-106b [66] and miR-196 [77] were upregulated in the intestinal epithelium of patients with active CD compared to controls. Schaefer et al. found an increase in miR-31, miR-101, and miR-146a levels in colon biopsy samples from $C D$ patients compared to healthy controls [88]. Further, miR-7 and miR-375 levels were decreased in the actively inflamed colonic mucosa of CD patients [88, 89].

Among the miRNAs included in the differential analysis of IBD colon tissues, miR-21, miR-31, and miR-141 are suggested to be valuable targets for the diagnosis of IBD. miR-21 is known to be an important factor in the pathogenesis of IBD as it modulates T-cell responses [90] and disrupts intercellular tight junctions in the intestinal epithelium [91]. miR-21 was found to be elevated in the mucosal tissue of both UC and $\mathrm{CD}[92,93]$, and the knockout of miR-21 in a mouse model of DSS-induced colitis inhibited the inflammatory response and increased survival rate, indicating the importance of miR-21 in IBD [52]. miR-31 was upregulated in the colon tissues of both UC and CD patients [88]; this miRNA is known to target IL-25 to stimulate the Th1/Th17-mediated pro-inflammatory molecular pathway in mouse colitis models [94]. miR-31 has also been suggested to be capable of distinguishing IBD from microscopic colitis, as its expression is elevated in IBD compared to microscopic colitis [95]. miR-141 inhibits CXCL5 and CXCL12 $\beta$, and its downregulation in IBD is expected to stimulate leukocyte recruitment and the consequent inflammatory response. A decrease in miR-141 was observed in the colon tissues of patients with UC [87].

Furthermore, useful findings have been obtained from the analysis of colon samples from patients with IBD. After acquiring rectal biopsy samples of UC and CD patients, Zham et al. found that miR-24 was increased in UC samples compared to CD samples, with a sensitivity of $83.3 \%$ and specificity of $85.7 \%$ as a diagnostic biomarker [96]. Jeremy et al. used matched colon biopsies from UC and CD patients to compare endoscopically involved regions with endoscopically uninvolved regions of each patient. miR-21, miR-31, miR-101, miR-142-3p, miR-142-5p, miR-155, miR-223, miR-375, and miR-494 levels were elevated in active 
CD lesions compared to inactive regions; miR-21, miR-101, miR-142-5p, miR-146a, miR-155, and miR-223 levels were higher in active UC lesions than in inactive regions [88]. Guo et al. compared inflamed and non-inflamed mucosal samples of the terminal ileum of active $C D$ patients and found that miR-124-3p and miR-192-5p levels were decreased, while miR-361-3p was upregulated in the inflamed mucosa of CD patients [97].

\section{miRNA signature from the peripheral blood of IBD patients}

As the acquisition of colon tissues requires a relatively invasive procedure compared to blood sampling, some studies have attempted to identify miRNA signatures from the peripheral blood of patients with active IBD. Paraskevi et al. reported increased levels of miR-16, miR-23a, miR-29a, miR-106a, miR-107, miR-126, miR-191, miR-199a-5p, miR-200c, miR-362-3p, and miR-532-3p in the blood of $\mathrm{CD}$ patients compared to healthy controls, and elevated levels of miR-16, miR-21, miR-28-5p, miR-151-5p, miR-155, and miR-199a-5p in the blood of UC patients compared to controls [98]. Wu et al. found increased levels of miR-199a-5p, miR-340*, miR-362-3p, miR-532-3p, and miRplus-E1271, and decreased levels of miR-149* and miRplus-F1065 in the blood of patients with active $\mathrm{CD}$ compared to controls [99]. The levels of miR-28-5p, miR-103-2*, miR-151-5p, miR-199a-5p, miR-340*, miR-362-3p, miR-532-3p, and miRplus-E1271 were increased, while that of miR-505* was decreased in the peripheral blood samples of active UC patients compared to healthy controls [99]. By measuring miRNAs from the platelet fraction, Duttagupta et al. found increased levels of miR-188-5p, miR-378, miR-422a, miR-500, miR-501-5p, miR-769-5p, and miR-874 in blood samples from UC patients compared to controls [100]. Schaefer et al. collected peripheral blood from UC and CD patients with normal controls and reported elevated levels of miR-19a, miR-101, miR-142-5p, miR-223, miR-375, and miR-494, and reduced levels of miR-21, miR-31, and miR-146a in the blood samples of UC patients compared to healthy controls; the levels of miR-101 and miR-375 were increased, while those of miR-21, miR-31, miR-146a, and miR-155 were decreased in the whole blood of CD patients compared to controls [88]. Zahm et al. reported elevated levels of miR-16, miR-20a, miR-21， miR-30e, miR-93, miR-106a, miR-140, miR-192, miR-195, and miR-484 in serum samples from pediatric $C D$ patients compared to normal controls [101].

Viennois et al. found that the levels of miR-29b-3p, miR-122-5p, miR-146a-3p, miR-150-5p,
miR-192-5p, miR-194-5p, and miR-375-3p were elevated in the serum of IL10 (-/-) mice, which distinguished peripheral blood samples from UC patients and healthy controls with $83.3 \%$ sensitivity [102]. Wu et al. reported that the serum levels of eight miRNAs (miR-28, miR-103-2*, miR-149*, miR-151, miR-340*, miR-505*, miR-532, and miR-plus-E1153) could be used to distinguish UC from CD [99]. Jensen et al. used reverse-transcription polymerase chain reaction (RT-PCR) to measure the miRNA levels in serum samples of CD patients and healthy controls. Thereafter, they proposed that a decrease in serum miR-16b could be used to diagnose CD, which yielded an area under the curve of $65 \%$ [103]. Zham et al. measured 11 miRNAs (miR-16, miR-20a, miR-21, miR-30e, miR-93, miR-106a, miR-140, miR-192, miR-195, miR-484, and let7b) in serum samples from $\mathrm{CD}$ patients and healthy controls and reported that their elevation served as a diagnostic marker for CD with a sensitivity of $70 \%-83 \%$ and specificity of $75 \%-$ $101 \%$. By performing miRNA microarray analysis of the saliva from UC patients, CD patients, and healthy controls, Schaefer et al. found increases in the levels of miR-21, miR-31, and miR-142-3p and a decrease in miR-142-5p levels in UC patients, while saliva samples from CD patients showed elevated miR-26a and miR-101 levels compared to controls [88].

Gallo et al. suggested that miRNAs in human saliva and serum samples mainly exist in the form of exosomes (encapsulated microvesicles) [104]. Vickers et al. presented evidence that miRNAs are transported by high-density lipoprotein (HDL) to recipient cells [105]. Exosomes and HDL can protect miRNAs from ribonucleases in the blood, which makes it easier to measure miRNA levels in serum samples.

\section{Role of miRNAs in the treatment of IBD}

As in vitro studies have confirmed the importance of miRNAs in the pathophysiology of IBD, some researchers have attempted to administered miRNAs in vivo to mice with experimental colitis. Intracolonic administration of miRNA mimic molecules and antagonists led to the overexpression and inhibition of miRNA expression, respectively [106].

\section{Nanoparticle-mediated approach}

Tian et al. found that the delivery of the miR-31 mimic into the colon of DSS-colitis mice alleviated the inflammatory response. miR-31 was bound to encapsulating microspheres and administered via enema, resulting in a slower loss of body weight and colon length, as well as increased epithelial proliferation and reduced inflammation, as confirmed by colon biopsy [107]. Neudecker et al. also reported 
the attenuation of occult bleeding, weight loss, and edema in DSS-colitis mice after intracolonic administration of synthetic murine miR-223 mimetic in nanoparticle lipid emulsion [108]. The intracolonic administration of miR-141 precursors in mice with TNBS-induced colitis resulted in a decrease in CXCL12 $\beta$ expression and leukocyte infiltration in the intestine [57]. miR-146b was also found to alleviate intestinal inflammation when administered into the peritoneum of DSS-induced colitis mice with expression vectors [74]. By using a mouse model of TNBS-induced colitis, Cheng et al. demonstrated that the intracolonic administration of pre-miR-19b can suppress the inflammatory process, with in vitro results suggesting that miR-19b inhibits suppressor of cytokine signaling 3 (SOCS3) to reduce inflammation of the intestinal epithelium in IBD [109]. miR-200b bound to microvesicles was administered to intestinal epithelial cells in vitro and the colon of TNBS-colitis mice in vivo; this administration resulted in the attenuation of intestinal fibrosis with suppression of TGF- $\beta 1$ mediated epithelium-to-mesenchyme transition [110].

Other routes of administration have been attempted to deliver miRNA mimic molecules to mice with colitis. Zhang et al. purified nanoparticles from edible ginger and found that their oral administration by colitis mice reduced the severity of colitis and promoted wound healing. The nanoparticles contained around 125 miRNAs. Further, the colon samples of mice administered the nanoparticles had a decrease in pro-inflammatory cytokines (TNF- $\alpha$, IL-6, and IL-1 $\beta$ ), increase in anti-inflammatory cytokines (IL-10 and IL-22), and increase in the proliferation of the epithelium based on the biopsy [111]. Fukata et al. attempted to intravenously administer miR-29a-3p and miR-29b-3p bound to supercarbonate apatite. These researchers reported a decrease in the inflammatory response in DSS-colitis mice and revealed that the subcutaneous injection of miR-29b bound to supercarbonate apatite inhibited the immune response by targeting $\mathrm{CD} 11 \mathrm{c}^{+}$dendritic cells in a DSS-colitis model [112].

\section{Conclusion}

IBD is a multifactorial disease associated with environmental and genetic factors. Dysbiosis of commensal bacteria and breakdown of the intestinal barrier integrity are considered to be major factors in the development of IBD. However, recent studies have indicated that genetic factors, including miRNA dysregulation, play a major role in the pathophysiology of IBD. Numerous miRNAs participate in the complex regulatory system of intestinal inflammation. Further, the number of molecular interactions seems to be uncountable; however, recent findings suggest the targeting of certain miRNAs as clinical biomarkers or treatment options. Additional in vivo studies should be conducted to validate data from in vitro studies and assess the practicality of manipulating miRNA expression in IBD.

\section{Abbreviations}

AhR: aryl hydrocarbon receptor; Akt: protein kinase B; ATG5: autophagy related 5; ATG16L1: autophagy related 16 like 1; BECN1: Beclin 1; CD: Crohn's disease; $\mathrm{CD}^{+}$: cluster of differentiation; $\mathrm{CXCL}$ : C-X-C motif chemokine ligand; DSS: Dextran sulfate sodium; EGFR: epidermal growth factor receptor; FOXO3 or FOXO3a: Forkhead box class O3; GM-CSF: granulocyte-macrophage colony-stimulating factor; GSK3B: glycogen synthase kinase 3 beta; GWAS: Genome-wide association studies; HDL: high-density lipoprotein; IBD: inflammatory bowel disease; IFN: interferon; Ig-: immunoglobulin; IL-: interleukin-; IL-12/23p40: p40 subunit of IL-12 and IL-23; ILC: innate lymphoid cell; LC3-II: the lipid modified form of microtubule-associated protein 1A/1B-light chain 3; MIP2: macrophage inflammatory protein 2; miRNA, miR: microRNA; MLCK: myosin light chain kinase; NF-kB: nuclear factor-light-chain-enhancer of activated B cells; NK T cell: natural killer $\mathrm{T}$ cell; NOD2: nucleotide-binding oligomerization domain-containing protein 2; ORMDL3: ORMDL sphingolipid biosynthesis regulator 3; p-Akt: phosphor-protein kinase B; PI3K: phosphoinositide 3-kinase; PIP2: phosphatidylinositol 4,5-biphosphate; PMN: polymorphonuclear leukocyte; p-mTOR: phosphorylated mammalian target of rapamycin; PTEN: phosphatase and tensin homolog; PTK6: protein tyrosine kinase 6; RA: retinoic acid; REG3: Regenerating islet-derived protein 3; RISC: RNA-induced silencing complex; RT-PCR: reversetranscription polymerase chain reaction; SAA: serum amyloid A; SFB: segmented filamentous bacteria; SHIP-1: Src homology 2 domain containing inositol polyphosphate 5-phosphatase 1; SIAH2: Siah E3 ubiquitin protein ligase 2; SOCS3: suppressor of cytokine signaling 3; STAT: signal transducers and activators of transcription; SQSTM1: sequestosome 1; TAK1: transforming growth factor beta-activated kinase 1 ; TGF $\beta 1$ : transforming growth factor $\beta 1$; Th1: T helper type 1; Th2: T helper type 2; Th17: T helper type 17; TNBS: 2,4,6-trinitrobenzen sulfonic acid; TNF-a: tumor necrosis factor-a; Treg cell: regulatory T cell; UC: ulcerative colitis; UTR: untranslated region; XBP1: X-box binding protein 1; ZO: zonula occludens. 


\section{Acknowledgements}

\section{Contributions}

All authors made substantial contributions to all of the following: (1) conception and design of the study, data acquisition, or analysis and interpretation of data; (2) drafting or critical revision of the article for intellectual content; and (3) final approval of version to be submitted.

\section{Competing Interests}

The authors have declared that no competing interest exists.

\section{References}

1. Guan Q. A Comprehensive Review and Update on the Pathogenesis of Inflammatory Bowel Disease. J Immunol Res. 2019; 2019: 7247238.

2. Stidham RW, Higgins PDR. Colorectal Cancer in Inflammatory Bowel Disease. Clin Colon Rectal Surg. 2018; 31: 168-783.

3. Ng SC, Shi HY, Hamidi N, Underwood FE, Tang W, Benchimol EI, et al. Worldwide incidence and prevalence of inflammatory bowel disease in the 21st century: a systematic review of population-based studies. Lancet. 2018; 390: 2769-78.

4. Kaplan GG, Windsor JW. The four epidemiological stages in the global evolution of inflammatory bowel disease. Nat Rev Gastroenterol Hepatol. 2021; 18: 56-66.

5. Uniken Venema WT, Voskuil MD, Dijkstra G, Weersma RK, Festen EA. The genetic background of inflammatory bowel disease: from correlation to causality. J Pathol. 2017; 241: 146-58.

6. Ellinghaus D, Jostins L, Spain SL, Cortes A, Bethune J, Han B, et al. Analysis of five chronic inflammatory diseases identifies 27 new associations and highlights disease-specific patterns at shared loci. Nat Genet. 2016; 48: 510-8.

7. de Lange KM, Moutsianas L, Lee JC, Lamb CA, Luo Y, Kennedy NA, et al. Genome-wide association study implicates immune activation of multiple integrin genes in inflammatory bowel disease. Nat Genet. 2017; 49: 256-61.

8. Huang H, Fang M, Jostins L, Umićević Mirkov M, Boucher G, Anderson CA, et al. Fine-mapping inflammatory bowel disease loci to single-variant resolution. Nature. 2017; 547: 173-8.

9. Liu JZ, van Sommeren S, Huang $\mathrm{H}, \mathrm{Ng} \mathrm{SC}$, Alberts R, Takahashi A, et al. Association analyses identify 38 susceptibility loci for inflammatory bowel disease and highlight shared genetic risk across populations. Nat Genet. 2015; 47: 979-86

10. Park SC, Jeen YT. Genetic Studies of Inflammatory Bowel Disease-Focusing on Asian Patients. Cells. 2019; 8 .

11. Spehlmann ME, Begun AZ, Burghardt J, Lepage P, Raedler A, Schreiber S. Epidemiology of inflammatory bowel disease in a German twin cohort: results of a nationwide study. Inflamm Bowel Dis. 2008; 14: 968-76.

12. Halme L, Paavola-Sakki P, Turunen U, Lappalainen M, Farkkila M, Kontula K. Family and twin studies in inflammatory bowel disease. World J Gastroenterol. 2006; 12: 3668-72.

13. Soroosh A, Koutsioumpa M, Pothoulakis C, Iliopoulos D. Functional role and therapeutic targeting of microRNAs in inflammatory bowel disease. Am J Physiol Gastrointest Liver Physiol. 2018; 314: G256-62.

14. O'Brien J, Hayder H, Zayed Y, Peng C. Overview of MicroRNA Biogenesis, Mechanisms of Actions, and Circulation. Front Endocrinol (Lausanne). 2018 ;9 : 402 .

15. Lu Q, Wu R, Zhao M, Garcia-Gomez A, Ballestar E. miRNAs as Therapeutic Targets in Inflammatory Disease. Trends Pharmacol Sci. 2019 ; 40: 853-65.

16. Vidigal JA, Ventura A. The biological functions of miRNAs: lessons from in vivo studies. Trends Cell Biol. 2015; 25: 137-47.

17. Lozupone CA, Stombaugh JI, Gordon JI, Jansson JK, Knight R. Diversity, stability and resilience of the human gut microbiota. Nature. $2012 \cdot 489 \cdot 220-30$.

18. Rooks MG, Garrett WS. Gut microbiota, metabolites and host immunity. Nat Rev Immunol. 2016; 16: 341-52.

19. Krause P, Morris V, Greenbaum JA, Park Y, Bjoerheden U, Mikulski Z, et al. IL-10-producing intestinal macrophages prevent excessive antibacterial innate immunity by limiting IL-23 synthesis. Nat Commun. $2015 ; 6: 7055$.

20. Zigmond E, Bernshtein B, Friedlander G, Walker CR, Yona S, Kim KW, et al. Macrophage-restricted interleukin-10 receptor deficiency, but not IL-10 deficiency, causes severe spontaneous colitis. Immunity. $2014 ;$ 40: 720-33.

21. Murdoch CC, Espenschied ST, Matty MA, Mueller O, Tobin DM, Rawls JF. Intestinal Serum amyloid A suppresses systemic neutrophil activation and bactericidal activity in response to microbiota colonization. PLoS Pathog. 2019 ; 15: e1007381.

22. Zhang G, Liu J, Wu L, Fan Y, Sun L, Qian F, et al. Elevated Expression of Serum Amyloid A 3 Protects Colon Epithelium Against Acute Injury Through
TLR2-Dependent Induction of Neutrophil IL-22 Expression in a Mouse Model of Colitis. Front Immunol. $2018 ; 9: 1503$.

23. Macpherson AJ, Yilmaz B, Limenitakis JP, Ganal-Vonarburg SC. IgA Function in Relation to the Intestinal Microbiota. Annu Rev Immunol. 2018; 36: 359-81.

24. Oliveira LM, Teixeira FME, Sato MN. Impact of Retinoic Acid on Immune Cells and Inflammatory Diseases. Mediators Inflamm. 2018; 2018:3067126.

25. Grizotte-Lake M, Zhong G, Duncan K, Kirkwood J, Iyer N, Smolenski I, et al. Commensals Suppress Intestinal Epithelial Cell Retinoic Acid Synthesis to Regulate Interleukin-22 Activity and Prevent Microbial Dysbiosis. Immunity. 2018 ; 49: 1103-15.e6.

26. Pernomian L, Duarte-Silva M, de Barros Cardoso CR. The Aryl Hydrocarbon Receptor (AHR) as a Potential Target for the Control of Intestinal Inflammation: Insights from an Immune and Bacteria Sensor Receptor. Clin Rev Allergy Immunol. 2020; 59: 382-90.

27. Schanz O, Chijiiwa R, Cengiz SC, Majlesain Y, Weighardt H, Takeyama H, et al.. Dietary AhR Ligands Regulate AhRR Expression in Intestinal Immune Cells and Intestinal Microbiota Composition. Int J Mol Sci. 2020; 21: 3189.

28. Yu LC. Microbiota dysbiosis and barrier dysfunction in inflammatory bowel disease and colorectal cancers: exploring a common ground hypothesis. J Biomed Sci. $2018 ; 25: 79$.

29. Odenwald MA, Turner JR. The intestinal epithelial barrier: a therapeutic target? Nat Rev Gastroenterol Hepatol. 2017; 14: 9-21.

30. Pickert G, Neufert C, Leppkes M, Zheng Y, Wittkopf N, Warntjen M, et al. STAT3 links IL-22 signaling in intestinal epithelial cells to mucosal wound healing. J Exp Med. 2009; 206: 1465-72.

31. Kotredes KP, Thomas B, Gamero AM. The Protective Role of Type I Interferons in the Gastrointestinal Tract. Front Immunol. 2017; 8: 410

32. Shih VF, Cox J, Kljavin NM, Dengler HS, Reichelt M, Kumar P, et al. Homeostatic IL-23 receptor signaling limits Th17 response through IL-22-mediated containment of commensal microbiota. Proc Natl Acad Sci U S A. 2014; 111: 13942-7.

33. Coccia M, Harrison OJ, Schiering C, Asquith MJ, Becher B, Powrie F, et al. IL-1beta mediates chronic intestinal inflammation by promoting the accumulation of IL-17A secreting innate lymphoid cells and CD4(+) Th17 cells. J Exp Med. 2012; 209: 1595-609.

34. Zielinski CE, Mele F, Aschenbrenner D, Jarrossay D, Ronchi F, Gattorno M, et al. Pathogen-induced human TH17 cells produce IFN-gamma or IL-10 and are regulated by IL-1beta. Nature. 2012; 484: $514-8$.

35. Nowarski R, Jackson R, Gagliani N, de Zoete MR, Palm NW, Bailis W, et al. Epithelial IL-18 Equilibrium Controls Barrier Function in Colitis. Cell. 2015; 163: $1444-56$

36. Atreya R, Zimmer M, Bartsch B, Waldner MJ, Atreya I, Neumann H, et al. Antibodies against tumor necrosis factor (TNF) induce T-cell apoptosis in patients with inflammatory bowel diseases via TNF receptor 2 and intestinal CD14(+) macrophages. Gastroenterology. 2011; 141: 2026-38

37. Pott J, Kabat AM, Maloy KJ. Intestinal Epithelial Cell Autophagy Is Required to Protect against TNF-Induced Apoptosis during Chronic Colitis in Mice. Cell Host Microbe. 2018; 23: 191-202 e4.

38. Hunter CA, Jones SA. IL-6 as a keystone cytokine in health and disease. Nat Immunol. 2015; 16: 448-57.

39. Griseri T, McKenzie BS, Schiering C, Powrie F. Dysregulated hematopoietic stem and progenitor cell activity promotes interleukin-23-driven chronic intestinal inflammation. Immunity. 2012; 37: 1116-29.

40. Griseri T, Arnold IC, Pearson C, Krausgruber T, Schiering C, Franchini F, et al. Granulocyte Macrophage Colony-Stimulating Factor-Activated Eosinophils Promote Interleukin-23 Driven Chronic Colitis. Immunity. 2015; 43: 187-99.

41. Fan H, Wang A, Wang Y, Sun Y, Han J, Chen W, et al. Innate Lymphoid Cells: Regulators of Gut Barrier Function and Immune Homeostasis. J Immunol Res. $2019 \cdot 2019 \cdot 2525984$

42. Schaefer JS. MicroRNAs: how many in inflammatory bowel disease? Curr Opin Gastroenterol. 2016; 32: 258-66.

43. Ma TY, Boivin MA, Ye D, Pedram A, Said HM. Mechanism of TNF-\{alpha\} modulation of Caco-2 intestinal epithelial tight junction barrier: role of myosin light-chain kinase protein expression. Am J Physiol Gastrointest Liver Physiol. 2005; 288: G422-30.

44. Wang L, Zhang R, Chen J, Wu Q, Kuang Z. Baicalin Protects against TNF-alpha-Induced Injury by Down-Regulating miR-191a That Targets the Tight Junction Protein ZO-1 in IEC-6 Cells. Biol Pharm Bull. 2017; 40: 435-43.

45. Tang Y, Zhang L, Forsyth CB, Shaikh M, Song S, Keshavarzian A. The Role of miR-212 and iNOS in Alcohol-Induced Intestinal Barrier Dysfunction and Steatohepatitis. Alcohol Clin Exp Res. 2015; 39: 1632-41.

46. Zou T, Jaladanki SK, Liu L, Xiao L, Chung HK, Wang JY, et al. H19 Long Noncoding RNA Regulates Intestinal Epithelial Barrier Function via MicroRNA 675 by Interacting with RNA-Binding Protein HuR. Mol Cell Biol. 2016; 36: 1332-41.

47. Zhi X, Tao J, Li Z, Jiang B, Feng J, Yang L, et al. MiR-874 promotes intestinal barrier dysfunction through targeting AQP3 following intestinal ischemic injury. FEBS Lett. 2014; 588: 757-63.

48. Zhang B, Tian Y, Jiang P, Jiang Y, Li C, Liu T, et al. MicroRNA-122a Regulates Zonulin by Targeting EGFR in Intestinal Epithelial Dysfunction. Cell Physiol Biochem. 2017; 42: 848-58.

49. Zhang L, Shen J, Cheng J, Fan X. MicroRNA-21 regulates intestinal epithelial tight junction permeability. Cell Biochem Funct. 2015; 33: 235-40.

50. Shen Y, Zhou M, Yan J, Gong Z, Xiao Y, Zhang C, et al. miR-200b inhibits TNF-alpha-induced IL-8 secretion and tight junction disruption of intestinal 
epithelial cells in vitro. Am J Physiol Gastrointest Liver Physiol. 2017; 312: G123-G32.

51. Haines RJ, Beard RS, Jr., Eitner RA, Chen L, Wu MH. TNFalpha/IFNgamma Mediated Intestinal Epithelial Barrier Dysfunction Is Attenuated by MicroRNA-93 Downregulation of PTK6 in Mouse Colonic Epithelial Cells. PLoS One. 2016; 11: e0154351.

52. Shi C, Liang Y, Yang J, Xia Y, Chen H, Han H, et al. MicroRNA-21 knockout improve the survival rate in DSS induced fatal colitis through protecting against inflammation and tissue injury. PLoS One. 2013; 8: e66814.

53. Zhao Y, Ma T, Chen W, Chen Y, Li M, Ren L, et al. MicroRNA-124 Promotes Intestinal Inflammation by Targeting Aryl Hydrocarbon Receptor in Crohn's Disease. J Crohns Colitis. 2016; 10: 703-12.

54. Corridoni D, Arseneau KO, Cominelli F. Functional defects in NOD2 signaling in experimental and human Crohn disease. Gut Microbes. 2014; 5: 340-4.

55. Pierdomenico M, Cesi V, Cucchiara S, Vitali R, Prete E, Costanzo M, et al. NOD2 Is Regulated By Mir-320 in Physiological Conditions but this Control Is Altered in Inflamed Tissues of Patients with Inflammatory Bowel Disease. Inflamm Bowel Dis. 2016; 22: 315-26.

56. Wu W, He C, Liu C, Cao AT, Xue X, Evans-Marin HL, et al. miR-10a inhibits dendritic cell activation and Th1/Th17 cell immune responses in IBD. Gut. 2015; 64: 1755-64.

57. Huang Z, Shi T, Zhou Q, Shi S, Zhao R, Shi H, et al. miR-141 Regulates colonic leukocytic trafficking by targeting CXCL12beta during murine colitis and human Crohn's disease. Gut. 2014; 63: 1247-57.

58. Yang Z, Klionsky DJ. Eaten alive: a history of macroautophagy. Nat Cell Biol. 2010; 12: 814-22.

59. Lapaquette $\mathrm{P}$, Bringer MA, Darfeuille-Michaud A. Defects in autophagy favour adherent-invasive Escherichia coli persistence within macrophages leading to increased pro-inflammatory response. Cell Microbiol. 2012; 14: 791-807.

60. Wang S, Huang Y, Zhou C, Wu H, Zhao J, Wu L, et al. The Role of Autophagy and Related MicroRNAs in Inflammatory Bowel Disease. Gastroenterol Res Pract. 2018; 2018: 7565076.

61. Salem M, Ammitzboell M, Nys K, Seidelin JB, Nielsen OH. ATG16L1: A multifunctional susceptibility factor in Crohn disease. Autophagy. 2015; 11: 585-94.

62. Chen Y, Du J, Zhang Z, Liu T, Shi Y, Ge X, et al. MicroRNA-346 mediates tumor necrosis factor alpha-induced downregulation of gut epithelial vitamin D receptor in inflammatory bowel diseases. Inflamm Bowel Dis. 2014; 20: 1910-8.

63. Li M, Zhang S, Qiu Y, He Y, Chen B, Mao R, et al. Upregulation of miR-665 promotes apoptosis and colitis in inflammatory bowel disease by repressing the endoplasmic reticulum stress components XBP1 and ORMDL3. Cell Death Dis. 2017; 8: e2699.

64. Liu L, He J, Wei X, Wan G, Lao Y, Xu W, et al. MicroRNA-20a-mediated loss of autophagy contributes to breast tumorigenesis by promoting genomic damage and instability. Oncogene. 2017; 36: 5874-84.

65. Nguyen HT, Dalmasso G, Muller S, Carriere J, Seibold F, Darfeuille-Michaud A. Crohn's disease-associated adherent invasive Escherichia coli modulate levels of microRNAs in intestinal epithelial cells to reduce autophagy. Gastroenterology. 2014; 146: 508-19.

66. Lu C, Chen J, Xu HG, Zhou X, He Q, Li YL, et al. MIR106B and MIR93 prevent removal of bacteria from epithelial cells by disrupting ATG16L1-mediated autophagy. Gastroenterology. 2014; 146: 188-99.

67. Li N, Miao Y, Shan Y, Liu B, Li Y, Zhao L, et al. MiR-106b and miR-93 regulate cell progression by suppression of PTEN via PI3K/Akt pathway in breast cancer. Cell Death Dis. 2017; 8: e2796.

68. Zhai Z, Wu F, Dong F, Chuang AY, Messer JS, Boone DL, et al. Human autophagy gene ATG16L1 is post-transcriptionally regulated by MIR142-3p. Autophagy. 2014; 10: 468-79.

69. Chen Y, Wang C, Liu Y, Tang L, Zheng M, Xu C, et al. miR-122 targets NOD2 to decrease intestinal epithelial cell injury in Crohn's disease. Biochem Biophys Res Commun. 2013; 438: 133-9.

70. Chuang AY, Chuang JC, Zhai Z, Wu F, Kwon JH. NOD2 expression is regulated by microRNAs in colonic epithelial HCT116 cells. Inflamm Bowel Dis. 2014; 20: 126-35.

71. Wang Y, Zhang X, Tang W, Lin Z, Xu L, Dong R, et al. miR-130a upregulates mTOR pathway by targeting TSC1 and is transactivated by NF-kappaB in high-grade serous ovarian carcinoma. Cell Death Differ. 2017; 24: 2089-100

72. Kim HY, Kwon HY, Ha Thi HT, Lee HJ, Kim GI, Hahm KB, et al. MicroRNA-132 and microRNA-223 control positive feedback circuit by regulating FOXO3a in inflammatory bowel disease. J Gastroenterol Hepatol. 2016; 31: 1727-35.

73. Birnie KA, Yip YY, Ng DC, Kirschner MB, Reid G, Prele CM, et al. Loss of miR-223 and JNK Signaling Contribute to Elevated Stathmin in Malignant Pleural Mesothelioma. Mol Cancer Res. 2015; 13: 1106-18.

74. Nata T, Fujiya M, Ueno N, Moriichi K, Konishi H, Tanabe H, et al. MicroRNA-146b improves intestinal injury in mouse colitis by activating nuclear factor-kappaB and improving epithelial barrier function. J Gene Med. 2013; 15: 249-60.

75. Rouquette-Jazdanian AK, Kortum RL, Li W, Merrill RK, Nguyen PH, Samelson LE, et al. miR-155 Controls Lymphoproliferation in LAT Mutant Mice by Restraining T-Cell Apoptosis via SHIP-1/mTOR and PAK1/FOXO3/BIM Pathways. PLoS One. 2015; 10: e0131823.
76. Huang X, Shen $Y$, Liu M, Bi C, Jiang C, Iqbal J, et al. Quantitative proteomics reveals that miR-155 regulates the PI3K-AKT pathway in diffuse large B-cell lymphoma. Am J Pathol. 2012; 181: 26-33.

77. Brest $\mathrm{P}$, Lapaquette $\mathrm{P}$, Souidi $\mathrm{M}$, Lebrigand $\mathrm{K}$, Cesaro A, Vouret-Craviari V, et al. A synonymous variant in IRGM alters a binding site for miR-196 and causes deregulation of IRGM-dependent xenophagy in Crohn's disease. Nat Genet. 2011; 43: 242-5.

78. Melmed GY, Siegel CA, Spiegel BM, Allen JI, Cima R, Colombel JF, et al. Quality indicators for inflammatory bowel disease: development of process and outcome measures. Inflamm Bowel Dis. 2013; 19: 662-8.

79. Canavese G, Villanacci V, Sapino A, Rocca R, Daperno M, Suriani R, et al. The diagnosis of inflammatory bowel disease is often unsupported in clinical practice. Dig Liver Dis. 2015; 47: 20-3.

80. Wu F, Zikusoka M, Trindade A, Dassopoulos T, Harris ML, Bayless TM, et al. MicroRNAs are differentially expressed in ulcerative colitis and alter expression of macrophage inflammatory peptide-2 alpha. Gastroenterology. 2008; 135: 1624-35 e24.

81. Fasseu M, Treton X, Guichard C, Pedruzzi E, Cazals-Hatem D, Richard C, et al. Identification of restricted subsets of mature microRNA abnormally expressed in inactive colonic mucosa of patients with inflammatory bowel disease. PLoS One. 2010; 5 .

82. Takagi T, Naito Y, Mizushima K, Hirata I, Yagi N, Tomatsuri N, et al. Increased expression of microRNA in the inflamed colonic mucosa of patients with active ulcerative colitis. J Gastroenterol Hepatol. 2010; 25 Suppl 1: S129-33.

83. Feng X, Wang H, Ye S, Guan J, Tan W, Cheng S, et al. Up-regulation of microRNA-126 may contribute to pathogenesis of ulcerative colitis via regulating NF-kappaB inhibitor IkappaBalpha. PLoS One. 2012; 7: e52782.

84. Bian Z, Li L, Cui J, Zhang H, Liu Y, Zhang CY, et al. Role of miR-150-targeting $\mathrm{c}-\mathrm{Myb}$ in colonic epithelial disruption during dextran sulphate sodium-induced murine experimental colitis and human ulcerative colitis. J Pathol. 2011; 225: 544-53.

85. Lin J, Welker NC, Zhao Z, Li Y, Zhang J, Reuss SA, et al. Novel specific microRNA biomarkers in idiopathic inflammatory bowel disease unrelated to disease activity. Mod Pathol. 2014; 27: 602-8.

86. Coskun M, Bjerrum JT, Seidelin JB, Troelsen JT, Olsen J, Nielsen OH. miR-20b, miR-98, miR-125b-1*, and let-7e* as new potential diagnostic biomarkers in ulcerative colitis. World J Gastroenterol. 2013; 19: 4289-99.

87. Cai M, Chen S, Hu W. MicroRNA-141 Is Involved in Ulcerative Colitis Pathogenesis via Aiming at CXCL5. J Interferon Cytokine Res. 2017; 37: 415-20.

88. Schaefer JS, Attumi T, Opekun AR, Abraham B, Hou J, Shelby H, et al. MicroRNA signatures differentiate Crohn's disease from ulcerative colitis. BMC Immunol. 2015; 16: 5.

89. Nguyen HT, Dalmasso G, Yan Y, Laroui H, Dahan S, Mayer L, et al. MicroRNA-7 modulates CD98 expression during intestinal epithelial cell differentiation. J Biol Chem. 2010; 285: 1479-89.

90. Ando Y, Mazzurana L, Forkel M, Okazaki K, Aoi M, Schmidt PT, et al. Downregulation of MicroRNA-21 in Colonic CD3+ T Cells in UC Remission. Inflamm Bowel Dis. 2016; 22: 2788-93.

91. Yang Y, Ma Y, Shi C, Chen H, Zhang H, Chen N, et al. Overexpression of miR-21 in patients with ulcerative colitis impairs intestinal epithelial barrier function through targeting the Rho GTPase RhoB. Biochem Biophys Res Commun. 2013; 434: 746-52.

92. Thorlacius-Ussing G, Schnack Nielsen B, Andersen V, Holmstrom K, Pedersen AE. Expression and Localization of miR-21 and miR-126 in Mucosal Tissue from Patients with Inflammatory Bowel Disease. Inflamm Bowel Dis. 2017; 23 : 739-52.

93. Wu F, Zhang S, Dassopoulos T, Harris ML, Bayless TM, Meltzer SJ, et al. Identification of microRNAs associated with ileal and colonic Crohn's disease. Inflamm Bowel Dis. 2010; 16: 1729-38.

94. Shi T, Xie Y, Fu Y, Zhou Q, Ma Z, Ma J, et al. The signaling axis of microRNA-31/interleukin-25 regulates Th1/Th17-mediated inflammation response in colitis. Mucosal Immunol. 2017; 10: 983-95.

95. Zhang C, Zhao Z, Osman H, Watson R, Nalbantoglu I, Lin J. Differential expression of miR-31 between inflammatory bowel disease and microscopic colitis. Microrna. 2014; 3: 155-9.

96. Zahm AM, Hand NJ, Tsoucas DM, Le Guen CL, Baldassano RN, Friedman JR. Rectal microRNAs are perturbed in pediatric inflammatory bowel disease of the colon. J Crohns Colitis. 2014; 8: 1108-17.

97. Guo Z, Wu R, Gong J, Zhu W, Li Y, Wang Z, et al. Altered microRNA expression in inflamed and non-inflamed terminal ileal mucosa of adult patients with active Crohn's disease. J Gastroenterol Hepatol. 2015; 30: 109-16.

98. Paraskevi A, Theodoropoulos G, Papaconstantinou I, Mantzaris G, Nikiteas N, Gazouli M. Circulating MicroRNA in inflammatory bowel disease. J Crohns Colitis. 2012; 6: 900-4.

99. Wu F, Guo NJ, Tian H, Marohn M, Gearhart S, Bayless TM, et al. Peripheral blood microRNAs distinguish active ulcerative colitis and Crohn's disease. Inflamm Bowel Dis. 2011; 17: 241-50.

100. Duttagupta R, DiRienzo S, Jiang R, Bowers J, Gollub J, Kao J, et al. Genome-wide maps of circulating miRNA biomarkers for ulcerative colitis. PLoS One. 2012; 7: e31241.

101. Zahm AM, Thayu M, Hand NJ, Horner A, Leonard MB, Friedman JR Circulating microRNA is a biomarker of pediatric Crohn disease. J Pediatr Gastroenterol Nutr. 2011; 53: 26-33. 
102. Viennois E, Zhao Y, Han MK, Xiao B, Zhang M, Prasad M, et al. Serum miRNA signature diagnoses and discriminates murine colitis subtypes and predicts ulcerative colitis in humans. Sci Rep. 2017; 7: 2520.

103. Jensen MD, Andersen RF, Christensen H, Nathan T, Kjeldsen J, Madsen JS. Circulating microRNAs as biomarkers of adult Crohn's disease. Eur J Gastroenterol Hepatol. 2015; 27: 1038-44.

104. Gallo A, Tandon M, Alevizos I, Illei GG. The majority of microRNAs detectable in serum and saliva is concentrated in exosomes. PLoS One. 2012; 7: e30679.

105. Vickers KC, Palmisano BT, Shoucri BM, Shamburek RD, Remaley AT. MicroRNAs are transported in plasma and delivered to recipient cells by high-density lipoproteins. Nat Cell Biol. 2011; 13: 423-33.

106. Chen WX, Ren LH, Shi RH. Implication of miRNAs for inflammatory bowel disease treatment: Systematic review. World J Gastrointest Pathophysiol. 2014; 5: 63-70.

107. Tian Y, Xu J, Li Y, Zhao R, Du S, Lv C, et al. MicroRNA-31 Reduces Inflammatory Signaling and Promotes Regeneration in Colon Epithelium, and Delivery of Mimics in Microspheres Reduces Colitis in Mice. Gastroenterology. 2019; 156: 2281-96 e6.

108. Neudecker V, Haneklaus M, Jensen O, Khailova L, Masterson JC, Tye H, et al. Myeloid-derived miR-223 regulates intestinal inflammation via repression of the NLRP3 inflammasome. J Exp Med. 2017; 214: 1737-52.

109. Cheng X, Zhang X, Su J, Zhang Y, Zhou W, Zhou J, et al. miR-19b downregulates intestinal SOCS3 to reduce intestinal inflammation in Crohn's disease. Sci Rep. 2015; 5: 10397.

110. Yang J, Zhou CZ, Zhu R, Fan H, Liu XX, Duan XY, et al. miR-200b-containing microvesicles attenuate experimental colitis associated intestinal fibrosis by inhibiting epithelial-mesenchymal transition. J Gastroenterol Hepatol. 2017; 32: 1966-74.

111. Zhang M, Viennois E, Prasad M, Zhang Y, Wang L, Zhang Z, et al. Edible ginger-derived nanoparticles: A novel therapeutic approach for the prevention and treatment of inflammatory bowel disease and colitis-associated cancer. Biomaterials. 2016; 101: 321-40.

112. Fukata T, Mizushima T, Nishimura J, Okuzaki D, Wu X, Hirose H, et al. The Supercarbonate Apatite-MicroRNA Complex Inhibits Dextran Sodium Sulfate-Induced Colitis. Mol Ther Nucleic Acids. 2018; 12: 658-71. 